Published in final edited form as:

J Am Chem Soc. 2016 July 27; 138(29): 9205-9211. doi:10.1021/jacs.6b04481.

\title{
Fidelity and Promiscuity of a Mycobacterial Glycosyltransferase
}

\author{
Kenzo Yamatsugu ${ }^{\dagger} \S$, Rebecca A. Splain $\dagger, \int$, and Laura L. Kiessling ${ }^{\dagger, \ddagger},{ }^{*}$ \\ tDepartment of Chemistry, University of Wisconsin-Madison, 1101 University Ave., Madison, WI \\ 53706 \\ ‡Department of Biochemistry, University of Wisconsin-Madison, 433 Babcock Dr., Madison, WI \\ 53706
}

\begin{abstract}
Members of the mycobacterium genus cause devastating human diseases, including tuberculosis (TB). Mycobacterium tuberculosis can resist some antibiotics because of its durable and impermeable cell envelope. This barrier is assembled from saccharide building blocks not found in mammals, including galactofuranose (Galf). Within the cell envelope, Gal $f$ residues are linked together to afford an essential polysaccharide, termed the galactan. The formation of this polymer is catalyzed by the glycosyltransferase GlfT2, a processive carbohydrate polymerase, which generates a sequence-specific polysaccharide with alternating regioisomeric (1-5) and $\beta(1-6) \mathrm{Gal} f$ linkages. GlfT2 exhibits high fidelity in linkage formation, as it will terminate polymerization rather than deviate from its linkage pattern. These findings suggest that GlfT2 would prefer an acceptor with canonical $\beta(1-5)$ and $\beta(1-6)$ Gal $f$ sequence. To test this hypothesis, we devised a synthetic route to assemble oligosaccharides with natural and non-natural sequences. GlfT2 could elongate each of these acceptors, even those with non-natural linkage patterns. These data indicate that the glycosyltransferase is surprisingly promiscuous in its substrate preferences. However, GlfT2 did favor some substrates: it preferentially acted on those in which the lipid-bearing Gal $f$ residue was connected to the sequence by a $\beta(1-6)$ glycosidic linkage. The finding that the relative positioning of the lipid and the non-reducing end of the acceptor influences substrate selectivity is consistent with a role for the lipid in acceptor binding. The data also suggest that the fidelity of GlfT2 for generating alternating $\beta(1-5)$ and $\beta(1-6)$ pattern of Galf residues arises not from preferential substrate binding but during processive elongation. These observations suggest that inhibiting the action of GlfT2 will afford changes in cell wall structure.
\end{abstract}

\section{Graphical Abstract}

\footnotetext{
*Address correspondence to kiessling@chem.wisc.edu.

\$Current address: Pharmaceutical Sciences, University of Tokyo, 7-3-1, Hongo, Bunkyo-ku, Tokyo 113-0033, JAPAN

$\int$ Current address: API Chemistry, GlaxoSmithKline, King of Prussia, PA

The authors declare no competing financial interest.

Supporting Information

Routes employed for the syntheses of trisaccharide 5-7, experimental protocols for the preparation of all new compounds, and spectral data for all new compounds. Experimental procedures for protein production and enzyme assays are also included. The Supporting Information is available free of charge on the ACS Publications website.
} 


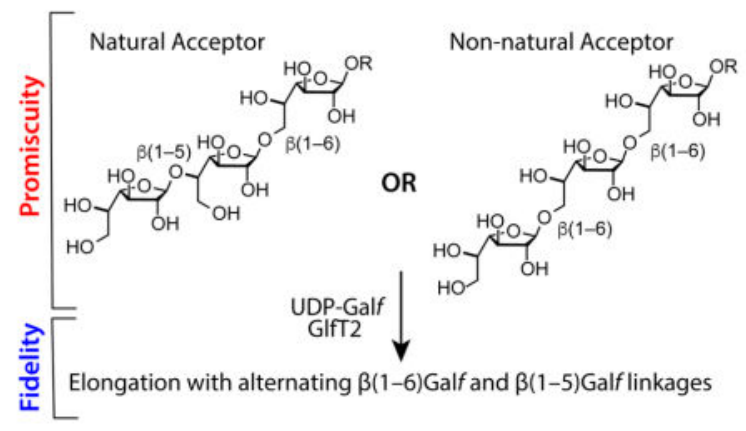

\section{Introduction}

Many glycans essential for microbial pathogenesis, virulence, or viability contain nonmammalian building blocks. Blocking the biosynthesis of these unique glycans could lead to new antibacterial therapies. The galactan of mycobacteria is composed of Gal $f$ residues, which are not found in mammalian glycans (Figure 1). The galactan is essential for mycobacterial viability, yet no enzymes that assemble the galactan are targeted by current antibiotics. Understanding the catalytic properties of galactan biosynthetic enzymes could lead to new strategies to target mycobacteria.

A key carbohydrate polymerase involved in galactan biosynthesis is GlfT2 (Figure 2). While most glycosyltransferases catalyze the formation of one type of glycosyl bond, some can generate multiple linkage types to yield oligosaccharides or polysaccharides of controlled sequence. ${ }^{1-4}$ Little is known, however, regarding the mechanism or substrate specificity of these multifunctional enzymes. GlfT2 is one such glycosyltransferase. In Mycobacterium tuberculosis, GlfT2 (EC 2.4.1.288), GlfT2 is one such glycosyltransferase. In Mycobacterium tuberculosis, GlfT2, which is encoded by the glfT2 (or Rv3808c) gene, is essential. ${ }^{5}$ GlfT2 promotes the formation of linear polysaccharides consisting of about 30 $40 \mathrm{Gal} f$ residues that are connected by alternating $\beta(1-5)$ and $\beta(1-6)$ linkages. ${ }^{6}$ The product polymer, the galactan, extends from the peptidoglycan and displays pendant arabinofuranose sequences that are linked ultimately to mycolic acids.

The natural glycosyl acceptor for GlfT2 is the tetrasacharide sequence D-Gal $f-\beta(1-5)-\mathrm{D}-$ Galf- $\beta(1-4)-\mathrm{L}-\mathrm{Rhaa}(1-3)-\mathrm{D}-\mathrm{GlcNAc}$ linked by a pyrophosphate to a polyprenol lipid (compound 1) ${ }^{7-9}$ This compound is generated through the action of the GlfT1. The ability of GlfT2 to generate polymers depends on the nature of the lipid substituent, ${ }^{10,11}$ but the glycosytransferase can readily process simple $\mathrm{diGal}_{f}$ motifs with lipophilic anomeric substituents. ${ }^{6,7,11-13}$ As a result, synthetic substrates have been used to probe the mechanism and products of GlfT2. GlfT2-catalyzed elongation results in polysaccharides similar in length to those isolated from mycobacteria. ${ }^{10,14}$ During elongation, GlfT2 catalyzes the formation of multiple $\mathrm{Gal}_{f}$ linkages before product release; it is a processive polymerase. ${ }^{10,14}$ Chain terminating nucleotide UDP-Gal $f$ derivatives have been used to reveal that GlfT2 faithfully generates alternating $\beta(1-5)$ and $\beta(1-6)$ Gal $f$ linkages. ${ }^{15,16}$ These studies revealed GlfT2's fidelity for assembly products with alternating regioisomeric linkages. The structure of GlfT2, determined by X-ray crystallography, indicates it can form 
a tetramer, with each monomer unit possessing a single active site. ${ }^{17}$ Site-directed mutagenesis studies support a model in which one active site facilitates formation of both glycosidic linkages. ${ }^{14}$ Sequence fidelity of the enzyme could be manifested by selectivity in substrate binding, in the process of elongation, or in both. To explore these possibilities, we sought to synthesize and test substrates whose sequences vary.

\section{Retrosynthesis}

The retrosynthesis of tetrasaccharide $\mathbf{8}$ illustrates our general strategy to assemble oligogalactofuranosides that vary in sequence (Figure 4). To maximize convergence, the tetrasaccharide was divided into disaccharide units 9 and 10. These building blocks could arise from monosaccharide precursors 11 and 12. Thioethylglycoside $\mathbf{1 2}$ was selected as a key intermediate for its ability to function either as an acceptor or as a donor. Specifically, the thioethyl anomeric group is sufficiently stable to allow a glycosylation reaction with more reactive donor $\mathbf{1 1} .^{18,19}$ The disaccharide 9 that would result from the first glycosylation reaction could be induced to undergo a subsequent reaction to build oligosaccharides.

Our oligosaccharide targets were predicated on findings that recombinant GlfT2 can extend acceptor mimics, such as $\mathbf{2}$, to afford polymers with lengths similar to those isolated from mycobacteria (Figure 3). ${ }^{11}$ To test the role of sequence in elongation, we focused on synthesizing trisaccharides $\mathbf{4}$ and $\mathbf{5}$ with naturally alternating linkages, trisaccharides $\mathbf{6}$ and $\mathbf{7}$ with non-natural consecutive linkages, and tetrasaccharide $\mathbf{8}$ with natural alternating linkages (Figure 3). The comparison of natural substrates (i.e., 4 and 5) to their non-natural (i.e., 6 and 7) counterparts would test whether GlfT2 can tolerate a "mistake" in the initial acceptor. In this way, we could assess the effect of different structural elements of the acceptor (internal and terminal glycosidic linkage) on the efficiency of polymerization by GlfT2.

We developed a divergent approach to produce oligogalactofuranoside acceptors with different sequences. The product oligosaccharides were assessed as substrates for GlfT2 by analyzing the efficiency of their elongation and the lengths of the product polysaccharides. Unexpectedly, substrates with perfectly alternating sequence were not preferred over those with "errors". It was the nature of the first glycosidic linkage following the lipid that had the largest influence on GlfT2-promoted glycosylation. The data indicate that the preferential formation of alternating $\beta(1-6) \mathrm{Gal} f$ then $\beta(1-5) \mathrm{Gal} f$ residues arises not from substrate binding but during catalytic processive polymerization.

Another feature of our strategy is that it uses intermediates that can be converted into acceptors with either a free O5 or O6 hydroxyl group. Specifically, the acetyl group from a compound such as $\mathbf{1 3}$ could be removed to yield an acceptor with C6 hydroxyl group (e.g., compound 12). Compounds with free C5 hydroxyl groups (e.g., 10) were envisioned to arise from benzoyl group migration from the C5 to the C6 hydroxyl group. Thus, oligosaccharide substrates could be assembled with either alternating or consecutive regioisomeric glycosidic linkages using only a small set of building blocks. 


\section{Route to oligosaccharide acceptors}

The aforementioned retrosynthetic strategy was applied to produce the target monosaccharide building blocks (Scheme 1). Both allyl glycoside nucleophile $\mathbf{1 6}$ with its C6 hydroxyl group free and $\mathbf{1 7}$ with its C5 hydroxyl group free were generated from acetoxy glycoside 15. ${ }^{11,20}$ Compound $\mathbf{1 5}$ was converted into the protected allyl glycoside. Acidmediated acetate removal from the $\mathrm{C} 6$ hydroxyl group provided 16, which is poised to form a 1,6-linkage. To obtain glycosidic acceptor $\mathbf{1 7}$ with a free C5 hydroxyl group, we sought to promote the migration of the benzoyl group from $\mathrm{O} 5$ position to $\mathrm{O} 6$ position. The desired transformation occurred readily when $\mathbf{1 6}$ was treated with triethylamine in methanol to afford $\mathbf{1 7}$ in $85 \%$ yield.

Similarly, compound $\mathbf{1 5}$ was efficiently converted to the $S$-ethyl thioglycosides $\mathbf{1 8}$ and $\mathbf{1 9 .}$ As anticipated, the $S$-ethyl anomeric group of $\mathbf{1 5}$ survives the acidic conditions for acetate removal. Compounds $\mathbf{1 8}$ and $\mathbf{1 9}$ can be used first as glycosyl acceptors, while the thioethyl group remains inert. The product of these reactions can function subsequently as an electrophile in glycosylation. By exploiting acetate group removal from O6 positions under mildly acidic conditions and the triethylamine-catalyzed benzoyl group migration, we could access each of the requisite monosaccharide building blocks.

We next examined the utility of these intermediates for the production of trisaccharide and tetrasaccharide substrates for GlfT2. The synthetic route to trisaccharide $\mathbf{4}$ is representative (Scheme 2). Glycosylation of $\mathbf{2 1}$ with acceptor $\mathbf{1 9}$ provided disaccharide 22. Only the thiazolinyl glycoside was activated with silver triflate, while the thioethyl functionality of acceptor 22 was undisturbed. ${ }^{18,19}$ When the disaccharide 22 was exposed to catalytic silver triflate and $\mathrm{N}$-iodosuccinimide (NIS) it underwent coupling to $\mathbf{1 6}$ to generate a trisaccharide with the natural alternating $\beta(1-6) \mathrm{Gal} f-\beta(1-5) \mathrm{Gal} f$ linkage pattern. We employed a cross metathesis reaction to append the 11-phenoxy-1-undecene moiety. Ester group removal provided the target trisaccharide acceptor 4. In an analogous manner, trisaccharides 5-7 were synthesized (See: Supporting Information).

Our route for trisaccharide assembly exploits common building blocks, yet it is divergent. It could be exploited construct onger oligomers, as illustrated by the production of tetrasaccharide $\mathbf{8}$ (Scheme 3). Compounds $\mathbf{1 6}$ and $\mathbf{2 3}$ were exposed to silver triflate to afford a disaccharide bearing a $\mathrm{C} 6$ acetate. Acetate removal and benzoyl group migration afforded glycosyl acceptor $\mathbf{2 4}$, which is poised for an $\mathrm{O} 5$ glycosylation reaction. Glycosylation with disaccharide $\mathbf{2 5}$ generated a tetrasaccharide that was transformed into target tetrasaccharide 8. We previously used a chemoenzymatic route to generate this compound but it afforded only small quantities $\left(1-5 \mathrm{mg}\right.$ ) of the tetrasaccharide. ${ }^{10}$ The synthetic route described herein is robust and scalable. Its use of orthogonally activatable thioglycosides and the application of the mild benzoyl group migration strategy to synthesize both O5 and O6 glycosylation acceptors enabled production of the tetrasaccharide glycolipid $\mathbf{8}$ in quantities sufficient to characterize GlfT2 elongation (vide infra). 


\section{Oligosaccharide elongation catalyzed by GIfT2}

We evaluated trisaccharides 4-7 and tetrasaccharide 8 in GlfT2-catalyzed polymerization reactions. To test their utility as substrates, recombinant GlfT2 from M. tuberculosis was mixed with the synthetic oligosaccharides and UDP-Galf. ${ }^{11}$ After 20 hours at room temperature, the crude enzymatic reaction mixtures were analyzed by MALDI-TOF mass spectrometry. The results were unexpected: All of the substrates were elongated including non-natural trisaccharides 6 and $\mathbf{7}$ (Figure 5). The polysaccharide products that result have as many Gal $f$ residues as found in the natural galactan (38-44 Gal $f$ residues). ${ }^{6}$ Given that GlfT2 processes all sequences, the data highlight the enzyme's promiscuity.

\section{Kinetic analyses of GIfT2 with oligosaccharide acceptors}

The intensity of peaks in the mass spectra suggests that some substrates (e.g., 4) are elongated more efficiently than others (e.g., 5). Surprisingly, however, the favorable substrates were not those that had "natural" alternating sequences. To quantify the activity of GlfT2 with different substrates, we used a continuous coupled assay to monitor the rates of GlfT2-catalyzed glycosylation of disaccharides 2 and 3, trisaccharides 4-7, and tetrasaccharide 8. Because GlfT2 produces UDP upon the addition of each Galfresidue to an acceptor, turnover can be assessed by linking UDP production to NADH oxidation. ${ }^{11,21-23}$ In agreement with previous results, ${ }^{11}$ GlfT2 exhibited a kinetic lag phase with disaccharide acceptors $\mathbf{2}$ and $\mathbf{3}$ (Figure 6). Though trisaccharide $\mathbf{7}$ exhibited a minor lag phase, none was observed with trisaccharides 4-6 nor with tetrasaccharide 8 . As the products of GlfT1 are tetrasaccharides and pentasaccharides, ${ }^{9}$ endogenous intermediates should be rapidly processed by GlfT2.

We monitored the initial rate of UDP production at different acceptor substrate concentrations to determine apparent $K_{\mathrm{m}}$ and $k_{\text {cat }}$ values for GIT2 (Table 1). Acceptors bearing anomeric lipids with longer chains bound more tightly to the enzyme and afforded higher catalyst turnover number than those with an octyl glycoside at the reducing end. ${ }^{13}$ These results are consistent with the influence of the anomeric lipid substitutent on acceptor elongation by GlfT2.

The specific saccharide sequence of each compound influenced its ability to serve as an acceptor. Surprisingly, the differences were not based on whether the acceptor had a natural or non-natural sequence. For example, a large difference in the kinetic parameters for trisaccharides $\mathbf{4}$ and $\mathbf{5}$ was observed, but both have the natural alternating linkages. Specifically, trisaccharide $\mathbf{4}$ with a $\beta(1-6)$ glycosidic linkage at the reducing end (i.e., near the lipid) had more favorable apparent $K_{\mathrm{m}}$ values $(13 \pm 1 \mu \mathrm{M})$ and higher catalyst turnover $\left(k_{\text {cat }}\right.$ of $3.7 \pm 0.1 \mathrm{~s}^{-1}$ ) than the trisaccharide 5 wiith a $\beta(1-5)$ glycosidic linkage at the reducing end (apparent $K_{\mathrm{m}}$ of $190 \pm 86 \mu \mathrm{M}$ and $k_{\text {cat }}$ of $0.72 \pm 0.15 \mathrm{~s}^{-1}$ ).

Comparison between $\mathbf{4}$ and $\mathbf{7}$ or between $\mathbf{5}$ and $\mathbf{6}$ further emphasizes the influence of the glycosidic linkage closest to the lipid (reducing end). Both acceptors 4 and 7 terminate with a $\beta(1-5)$ linkage, but 4 , with a reducing end $\beta(1-6)$ linkage exhibited 2-fold smaller apparent $K_{\mathrm{m}}$ and 4-fold higher $k_{\text {cat }}$ than did acceptor 7 with a $\beta(1-5)$ linkage at the reducing end. Similarly, acceptor 6 with the internal $\beta(1-6)$ linkage exhibited 5 -fold more favorable 
apparent $K_{\mathrm{m}}$ and 7-fold higher $k_{\text {cat }}$ than did acceptor 5 with the internal $\beta(1-5)$ linkage. These results indicate that the relative positioning of the acceptor lipid substituent impacts substrate binding to GlfT2 and subsequent catalytic turnover. A comparison of the $k_{\mathrm{cat}} / K_{\mathrm{m}}$ values for all of the oligosaccharide substrates indicates that the acceptors with a $\beta(1-6)$ near the reducing end $(\mathbf{4}, \mathbf{6}, \mathbf{8})$ are superior substrates to those with a corresponding $\beta(1-5)$ linkage $(5,7)$. Thus, the positioning or orientation of the saccharide relative to the anomeric lipid substituent is more important than whether the sequence alternates.

\section{DISCUSSION}

Data from our group and others indicate that GlfT2 has high fidelity for generating alternating $\beta(1-5)$ and $\beta(1-6)$ linkages. ${ }^{15,16}$ The alternating linkages could be required for subsequent steps in cell envelope biosynthesis or the stability of the cell envelope itself. We therefore examined whether GlfT2 can process acceptors with "mistakes" in the acceptor sequence. To address this issue, we designed oligogalactofuranose glycolipids 4-8 with different saccharide sequences (Figure 2). Synthetic trisaccharide acceptor substrates for GlfT2 have been studied previously, but the reported products of GlfT2-catalyzed reactions did not approach the length of the native galactan. ${ }^{13}$ Moreover, previous investigations focused on substrates with natural alternating glycosidic linkages. ${ }^{13}$ We therefore developed a divergent approach to synthesize GlfT2 substrates that vary in sequence.

Access to the oligogalactofuranose glycolipids was attained by using orthogonally activatable glycosyl donors and a facile method to quickly afford acceptors with different sequences of $\beta(1-6)$ and $\beta(1-5)$ Gal $f$ linkages. One key to the strategy was its reliance on $S$ thiazolinyl and $S$-ethyl glycosides as glycosyl donors with either high or moderate reactivity. ${ }^{18,19}$ The stability of thioglycosides is an advantage compared to more acid sensitive, reactive intermediates, such as trichloroacetimidate donors. ${ }^{20,24}$ The second important feature of our strategy was the migration of the benzoyl group to the least hindered $\mathrm{O} 6$ position.

Acyl group migration is often viewed as a problem in glycan synthesis, but our route exploits it. A previous synthetic approach to galactofuranose derivatives employed a selective protecting group strategy to afford an acceptor nucleophile with free C5 hydroxyl group. The key intermediate was synthesized by non-selective removal of primary acetyl group from C6 position, which resulted in a concomitant benzoyl group migration in low to moderate yield. ${ }^{20,24}$ We found a base-catalyzed benzoyl group migration provided the means to distinguish between adjacent hydroxyl groups in different steric environments. The orthogonal glycosyl donors and the acyl group migration are the two central strategic features that made it possible to rapidly access acceptors with either natural or non-natural sequences to evaluate with GlfT2. Because the synthetic route outlined relies on a small number of reactions that can be repeated iteratively, we anticipate that it could be used to access longer oligosaccharides with any desired combination of glycosidic linkage pattern.

It is striking that each substrate tested, trisaccharides 4-7 and tetrasaccharide 8, was elongated by GlfT2. The length of the product carbohydrate polymers was similar to that of the endogenous mycobacterial galactan (30-40 Gal $f$ residues). ${ }^{6}$ Acceptor 4 afforded slightly 
shorter galactan than the other three trisaccharide substrates. The observed truncated oligosaccharide could result from efficient consumption of the donor, which would limit the available quantity of UDP-Galf as the polymerization proceeds. ${ }^{6}$ We previously demonstrated that the presence and features of the lipid substituent of the initiating acceptor substrates influenced the product carbohydrate polymer length, and we proposed a tethering mechanism for length control by a template-independent polymerase. ${ }^{11}$ The findings shown herein that all the oligosaccharide acceptor substrates were elongated to the length of the galactan regardless of the different carbohydrate motifs lends additional support to the model. Our results also indicate that GlfT2 exhibits fidelity in the sequence generated during elongation, yet the carbohydrate polymerase is far more promiscuous in processing substrates of different sequences.

Previous studies demonstrated that GlfT2 can elongate disaccharide acceptors with an arabinose residue at the non-reducing end ${ }^{13}$ or even a glycolipid bearing a single Gal $f$ residue. ${ }^{20}$ We now show that trisaccharides with non-natural glycosidic linkages are elongated by GlfT2. Once GlfT2 begins elongation, its sequence fidelity is high. ${ }^{15}$ While this finding was unexpected, in mycobacterial cells, the only available Gal $f$ glycolipid initiators are those generated from the upstream galactofuranosyltransferase GlfT1. 7,8,25 This enzyme exhibits high specificity for either the native acceptor or structurally similar analogs. Thus, the high specificity of GlfT1 should ensure that GlfT2 can access the "correct" initial acceptor. The generation of polysaccharides with alternating linkages by GlfT2 appears to occur during processive elongation of the substrate. Specifically, as GlfT2 acts on the substrate, the growing polymer remains bound to the enzyme. ${ }^{10}$ In this way, the orientation of the nucleophilic nonreducing end of the growing polymer could be poised to generate the alternating linkages in the enzyme active site. ${ }^{14,17}$

We found previously that GlfT2 can act on both $\beta(1-6)$ - and $\beta(1-5)$-linked disaccharide acceptors $\mathbf{2}$ and $\mathbf{3}$ to form products with similar degrees of polymerization. ${ }^{20}$ There is, however, a significant difference in their initial reaction rate; $\beta$ (1-6)-linked disaccharide 2 has a reaction rate 4 -fold faster $(5.7 \pm 3.9 \mu \mathrm{M} / \mathrm{min})$ than that observed for $\beta(1-5)$-linked disaccharide $3(1.3 \pm 0.4 \mu \mathrm{M} / \mathrm{min}$, Figure 6$)$. The data indicate that the ability of an acceptor with a $\beta(1-6)$ linkage near the reducing end is a superior substrate for GlfT2 compared to a $\beta(1-5)$-linked acceptor. These results using either perfectly alternating or mismatched acceptors with $\beta(1-6)$ near the reducing end indicates that larger contribution to both acceptor binding and catalyst turnover than the glycosidic linkage at the non-reducing end. These observations are consistent with the saturation transfer difference (STD) NMR studies that indicate a reducing end $\mathrm{Gal} f$ residue in trisaccharide acceptors makes more intimate contacts with GlfT2 than a nonreducing end Gal $f$ residue. ${ }^{26}$ They suggest that the relative positioning of the lipid and the glycan is critical for elongation.

We previously found GlfT2 exhibited a kinetic lag phase with a disaccharide acceptor, while no such lag occurred with a chemoenzymatically synthesized tetrasaccharide. ${ }^{10}$ The data suggested that the lag phase arose from the lower affinity of the disaccharide acceptor for GlfT2. These findings led us to propose that GlfT2 has subsites, and oligosaccharide acceptors that fill the subsites would not exhibit a kinetic lag phase. This substrate subsite model also has been proposed for other carbohydrate polymerases. ${ }^{27-33}$ It was, however, 
unknown how many carbohydrate residues are necessary to fully occupy the subsites of GlfT2. Our results indicate that neither trisaccharides 4-6 nor tetrasaccharide $\mathbf{8}$ give rise to a kinetic lag phase. Kinetic analyses of trisaccharide $\mathbf{4}$ and tetrasaccharide $\mathbf{8}$ revealed that both have similar apparent $K_{\mathrm{m}}$ values, and additional Gal $f$ residues did not lead to large rate gains during the course of GlfT2-catalyzed glycosylation. This observation is interesting because the natural acceptor substrate for GlfT2 is a glycolipid composed of a tetrasaccharide or pentasaccharide generated by GlfT1. Thus, natural acceptor would fill the enzyme subsites, so that GIfT2 could efficiently elongate the initial acceptor.

We postulate that rapid processive elongation of endogenous acceptors by GlfT2 is inextricably correlated with high fidelity in formation of the alternating $\beta(1-5)$ and $\beta(1-6)$ regioisomeric linkages. Our finding that GlfT2 can process a variety of Galf-containing acceptors of varying sequence indicate that substrate binding is not responsible for sequence selectivity. It is in the processive formation of multiple glycosidic linkages that leads to carbohydrate polymer of defined sequence.

\section{CONCLUSION}

A robust and divergent synthetic approach has been developed to assemble lipid-modified oligogalactofuranose glycans. Two key features, orthogonally activatable thioglycosides and a benzoyl group migration strategy enabled the production of both alternating (natural) and non-alternating (non-natural) regioisomeric oligosaccharide acceptors for the mycobacterial galactofuranosyltransferase GlfT2. Polymerization and kinetic analyses of the synthetic acceptors revealed that the saccharide sequence of the initial acceptor influences glycosyltransferase elongation. All of the oligosaccharide acceptors were efficiently processed. These results support the tethering model previously proposed for the length control by a template-independent carbohydrate polymerase. ${ }^{11}$ The polymerization results revealed the sequence promiscuity of GlfT2 for its initial acceptor. Moreover, the results reveal that the nature of the glycosidic linkage closest to the reducing end of the acceptor makes a larger contribution to the efficiency of the catalysis by GlfT2. These observations provide further support for the importance of the lipid for catalysis. They also suggest that inhibitors that promote GlfT2 release of substrate could afford either shortened galactan or galactan in which deviations from the alternating $\beta(1-5)$ and $\beta(1-6)$ linkages occur. The possibility that such defects in the cell envelope would be detrimental provides impetus to seek GlfT2 inhibitors.

\section{Supplementary Material}

Refer to Web version on PubMed Central for supplementary material.

\section{Acknowledgments}

NIH is acknowledged for financial support (AI063596). K.Y. thanks JSPS Postdoctoral Fellowships for Research Abroad, and R.A.S. thanks American Chemical Society Division of Medicinal Chemistry Graduate Fellowship respectively for financial supports. All compound characterizations were performed at the University of WisconsinMadison Chemistry Instrument Center. The NMR facility is supported by the NSF (CHE-9208463), the Mass Spectrometry Facility by the NIH (NCRR 1S10RR024601-01) and NSF (CHE-9974839). We thank A. Justen, V. Winton, G. Kincaid, and D. Wesener for helpful comments on the manuscript. 


\section{References}

1. Whitfield C, Trent MS. Annu Rev Biochem. 2014; 83:99-128. [PubMed: 24580642]

2. Yother J. Annu Rev Microbiol. 2011; 65:563-81. [PubMed: 21721938]

3. Lombard V, Ramulu HG, Drula E, Coutinho PM, Henrissat B. Nucleic Acids Res. 2014; 42:D490D5. [PubMed: 24270786]

4. Cantarel BL, Coutinho PM, Rancurel C, Bernard T, Lombard V, Henrissat B. Nucleic Acids Res. 2009; 37:D233-D8. [PubMed: 18838391]

5. Sassetti CM, Boyd DH, Rubin EJ. Mol Microbiol. 2003; 48:77-84. [PubMed: 12657046]

6. Besra GS, Khoo KH, McNeil MR, Dell A, Morris HR, Brennan PJ. Biochemistry. 1995; 34:425766. [PubMed: 7703239]

7. Beláňová M, Dianišková P, Brennan PJ, Completo GC, Rose NL, Lowary TL, Mikušová K. J Bacteriol. 2008; 190:1141-5. [PubMed: 18055597]

8. Mikušová K, Beláňová M, Korduláková J, Honda K, McNeil MR, Mahapatra S, Crick DC, Brennan PJ. J Bacteriol. 2006; 188:6592-8. [PubMed: 16952951]

9. Martinez Farias MA, Kincaid VA, Annamalai VR, Kiessling LL. J Am Chem Soc. 2014; 136:84925. [PubMed: 24866828]

10. Levengood MR, Splain RA, Kiessling LL. J Am Chem Soc. 2011; 133:12758-66. [PubMed: 21739979]

11. May JF, Splain RA, Brotschi C, Kiessling LL. Proc Natl Acad Sci USA. 2009; 106:11851-6. [PubMed: 19571009]

12. Kremer L, Dover LG, Morehouse C, Hitchin P, Everett M, Morris HR, Dell A, Brennan PJ, McNeil MR, Flaherty C, Duncan K, Besra GS. J Biol Chem. 2001; 276:26430-40. [PubMed: 11304545]

13. Rose NL, Completo GC, Lin SJ, McNeil M, Palcic MM, Lowary TL. J Am Chem Soc. 2006; 128:6721-9. [PubMed: 16704275]

14. May JF, Levengood MR, Splain RA, Brown CD, Kiessling LL. Biochemistry. 2012; 51:1148-59. [PubMed: 22217153]

15. Brown CD, Rusek MS, Kiessling LL. J Am Chem Soc. 2012; 134:6552-5. [PubMed: 22458542]

16. Poulin MB, Zhou RK, Lowary TL. Org Biomol Chem. 2012; 10:4074-87. [PubMed: 22499274]

17. Wheatley RW, Zheng RB, Richards MR, Lowary TL, Ng KKS. J Biol Chem. 2012; 287:28132-43. [PubMed: 22707726]

18. Demchenko AV, Pornsuriyasak P, De Meo C, Malysheva NN. Angew Chem Int Ed. 2004; 43:3069-72.

19. Pornsuriyasak P, Demchenko AV. Chem Eur J. 2006; 12:6630-46. [PubMed: 16800023]

20. Splain RA, Kiessling LL. Biorg Med Chem. 2010; 18:3753-9.

21. Gosselin S, Alhussaini M, Streiff MB, Takabayashi K, Palcic MM. Anal Biochem. 1994; 220:92-7. [PubMed: 7978262]

22. Chen L, Men H, Ha S, Ye XY, Brunner L, Hu Y, Walker S. Biochemistry. 2002; 41:6824-33. [PubMed: 12022887]

23. Rose NL, Zheng RB, Pearcey J, Zhou R, Completo GC, Lowary TL. Carbohydr Res. 2008; 343:2130-9. [PubMed: 18423586]

24. Completo GC, Lowary TL. J Org Chem. 2008; 73:4513-25. [PubMed: 18489149]

25. Alderwick LJ, Dover LG, Veerapen N, Gurcha SS, Kremer L, Roper DL, Pathak AK, Reynolds RC, Besra GS. Protein Expr Purif. 2008; 58:332-41. [PubMed: 18248822]

26. Szczepina MG, Zheng RB, Completo GC, Lowary TL, Pinto BM. Biorg Med Chem. 2010; 18:5123-8.

27. Schwartz B, Markwalder JA, Seitz SP, Wang Y, Stein RL. Biochemistry. 2002; 41:12552-61. [PubMed: 12369847]

28. Chen L, Walker D, Sun B, Hu Y, Walker S, Kahne D. Proc Natl Acad Sci USA. 2003; 100:565863. [PubMed: 12714684]

29. Jing W, DeAngelis PL. J Biol Chem. 2004; 279:42345-9. [PubMed: 15299014]

30. Forsee WT, Cartee RT, Yother J. J Biol Chem. 2006; 281:6283-9. [PubMed: 16410247] 
31. Vionnet J, Vann WF. Glycobiology. 2007; 17:735-43. [PubMed: 17384120]

32. Barrett D, Wang TSA, Yuan Y, Zhang Y, Kahne D, Walker S. J Biol Chem. 2007; 282:31964-71. [PubMed: 17704540]

33. Wang TSA, Lupoli TJ, Sumida Y, Tsukamoto H, Wu Y, Rebets Y, Kahne DE, Walker S. J Am Chem Soc. 2011; 133:8528-30. [PubMed: 21568328] 


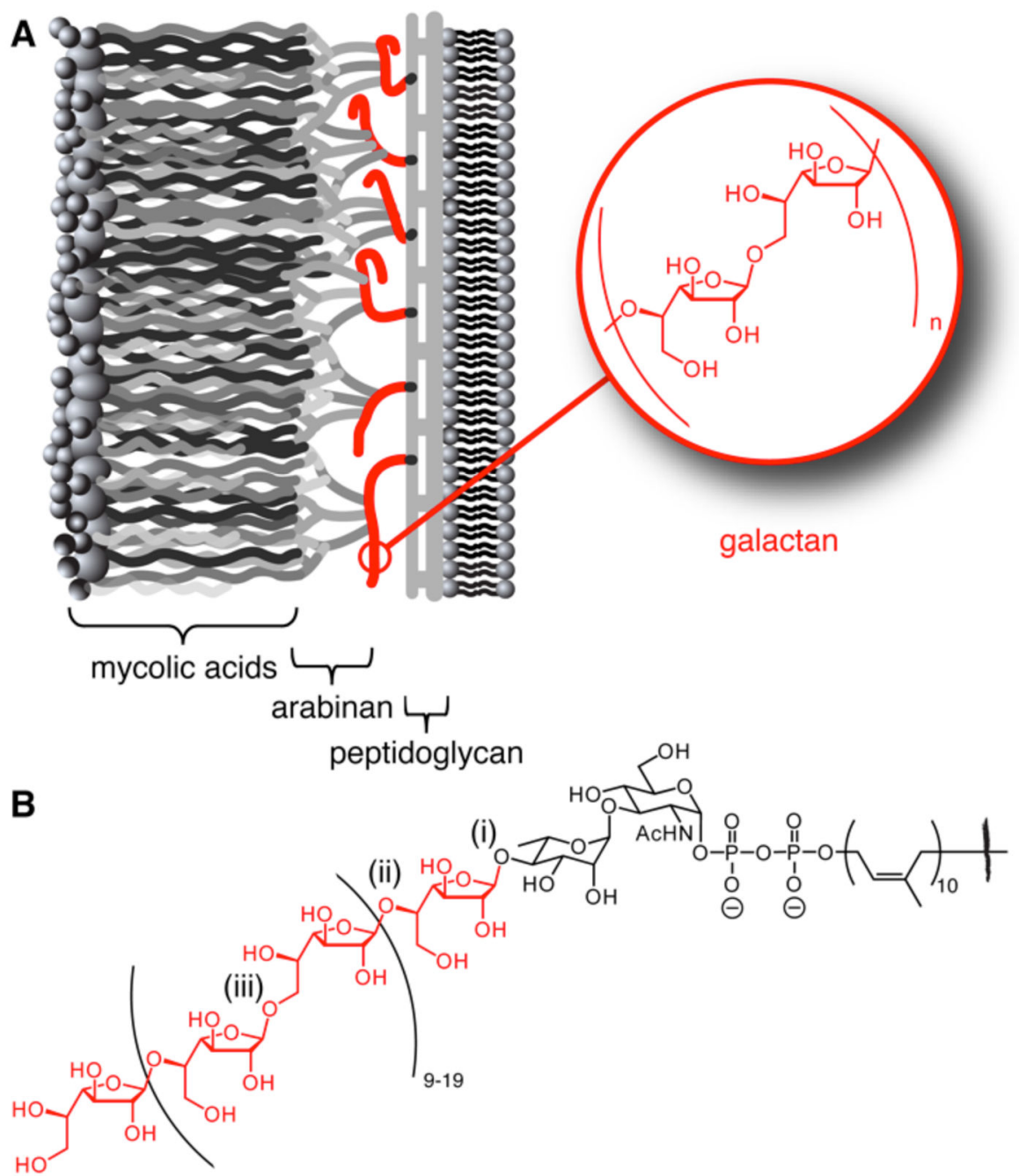

Figure 1.

The mycobacterial cell envelope contains an essential polymer composed of Galf residues. A. Schematic of the mycobacterial cell envelope showing that the galactan (red) links the mycolic acids to the peptidoglycan. B. Mycobacteria generate the lipid-linked carbohydrate polymer, which then is covalently linked to the peptidoglycan. 


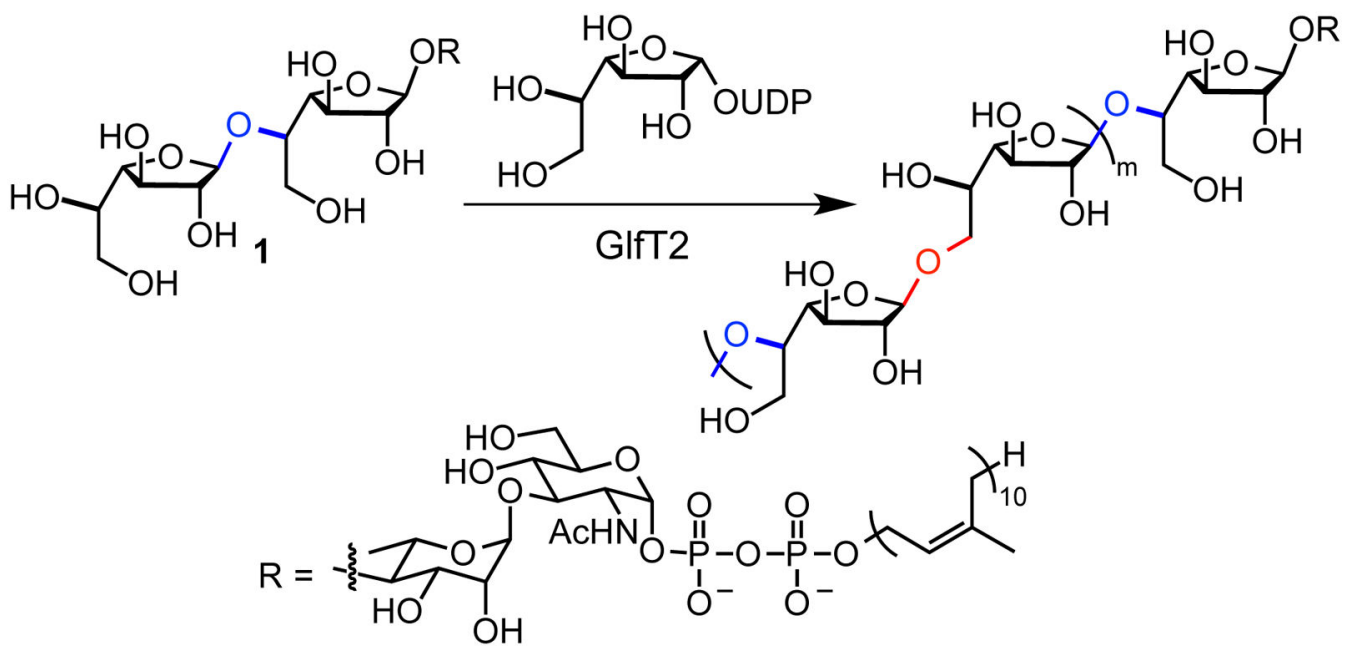

Figure 2.

GlfT2 mediates galactan formation by catalyzing the addition of 30-40 Gal $f$ residues to oligosaccharide-lipid precursor 1. GlfT2 is a bifunctional glycosyltransferase that can generate alternating Gal $f-\beta(1-5) \mathrm{Gal} f$ and Gal $f-\beta(1-6) \mathrm{Gal} f$ linkages. 

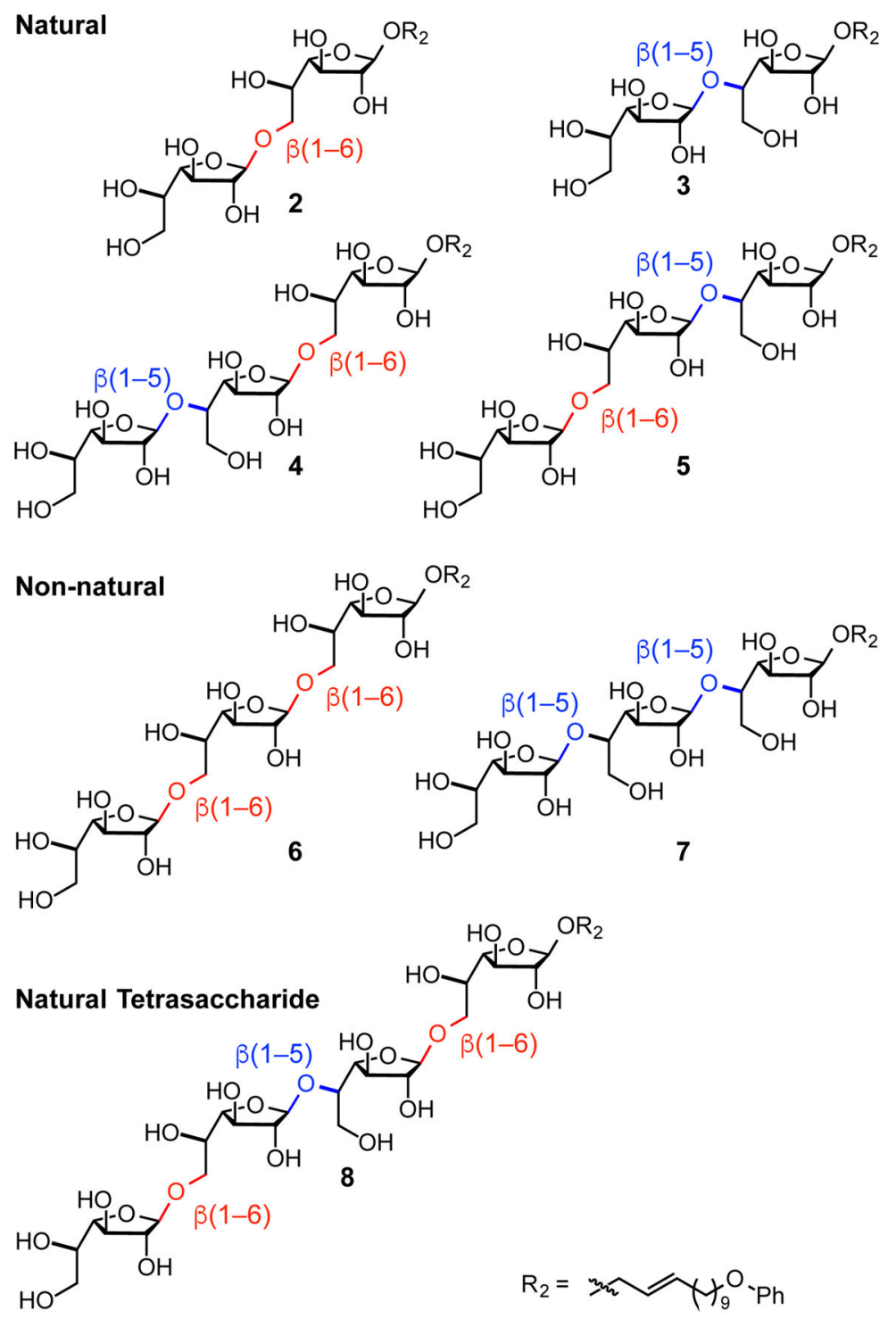

Figure 3.

Oligosaccharides synthesized to probe GlfT2 specificity. Trisaccharides 4 and 5 and tetrasaccharide 8 have natural alternating glycosidic linkages, while trisaccharides 6 and 7 have non-natural consecutive glycosidic linkages. 


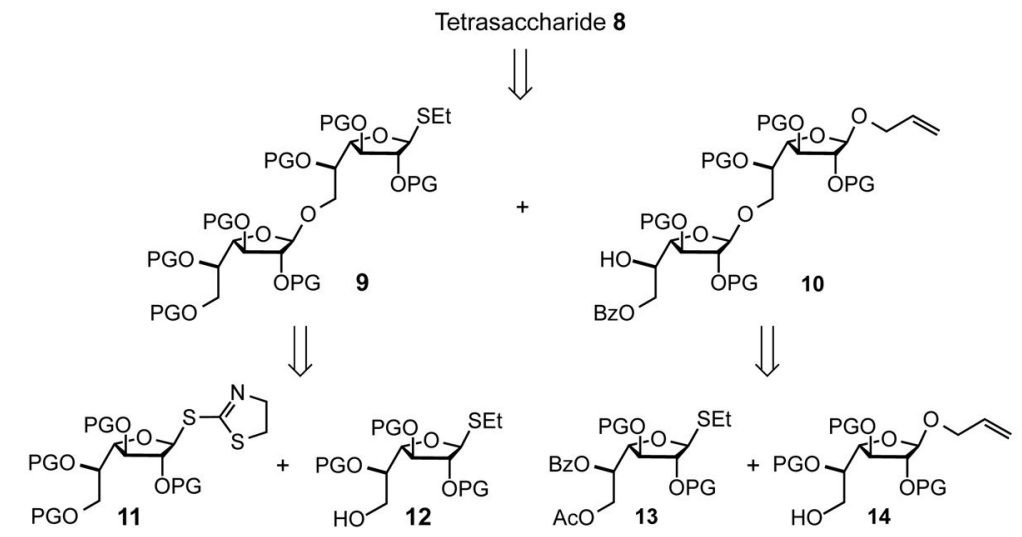

Figure 4.

Retrosynthetic analysis of tetrasccharide acceptor $\mathbf{8}$. The precursor for thioglycosides $\mathbf{1 1}$ and 12 can be activated for glycosylation under different conditions, and a benzoyl group migration strategy can be employed to access acceptors with either a C5 or a C6 hydroxyl group poised for glycosylation. 

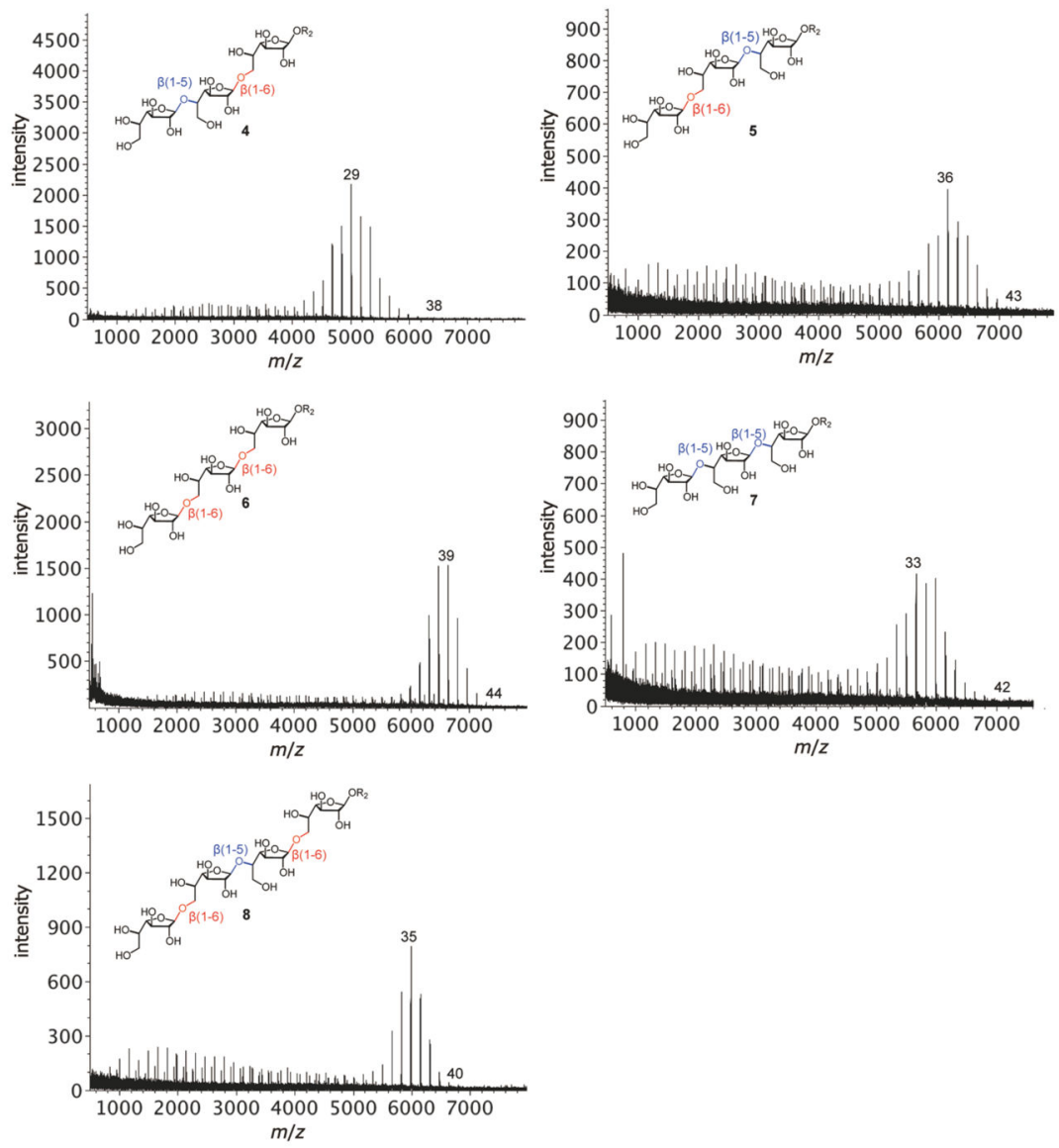

Figure 5.

MALDI-TOF mass spectra of GlfT2-catalyzed polymerization reactions with trisaccharide acceptors 4-7 and tetrasaccharide acceptor 8. Synthetic oligosaccharides were incubated at rt with GlfT2 in the presence of UDP-Galf. After $20 \mathrm{~h}$, the resulting mixtures were analyzed by MALDI-TOF mass spectrometry. Peaks that correspond to $\mathrm{m} / z$ values of $[\mathrm{M}+\mathrm{Na}]^{+}$are labeled with the value of $n$, which is the number of Gal $f$ residues in the product. 


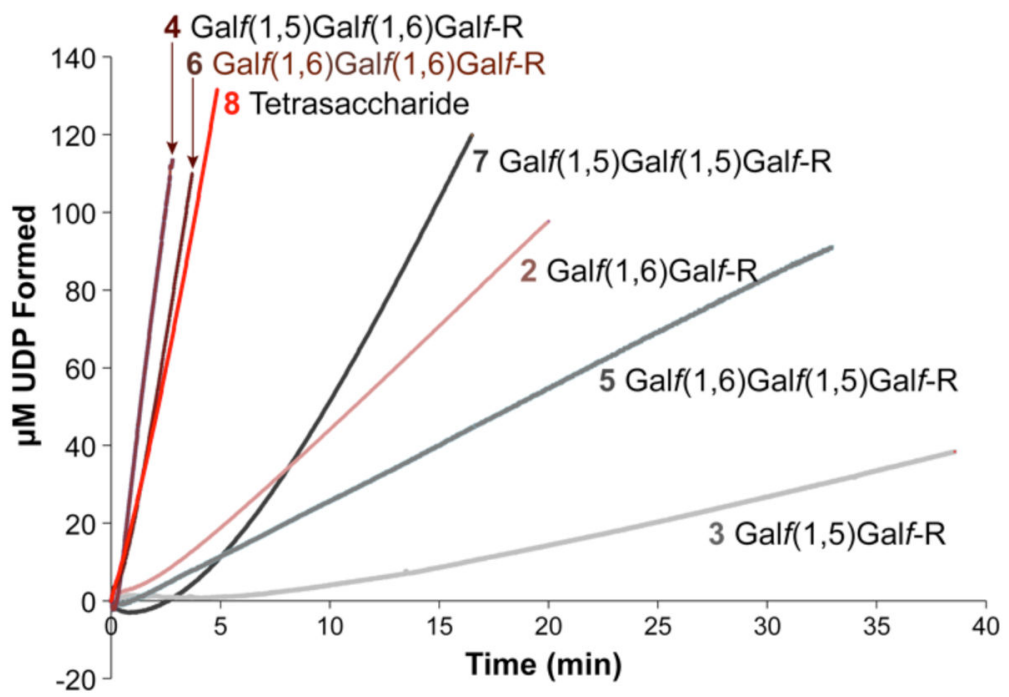

Figure 6.

Kinetics of GlfT2-catalyzed polymerization with oligosaccharide acceptors 2-8. GlfT2 turnover was monitored by coupling UDP production to NADH oxidation in a continuous assay. 


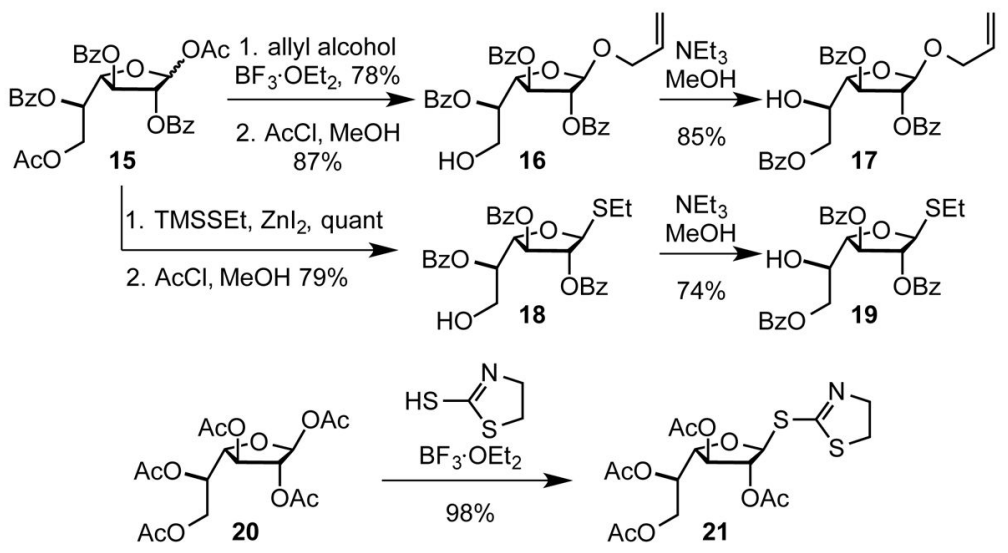

Scheme 1.

Synthesis of monosaccharide building blocks 


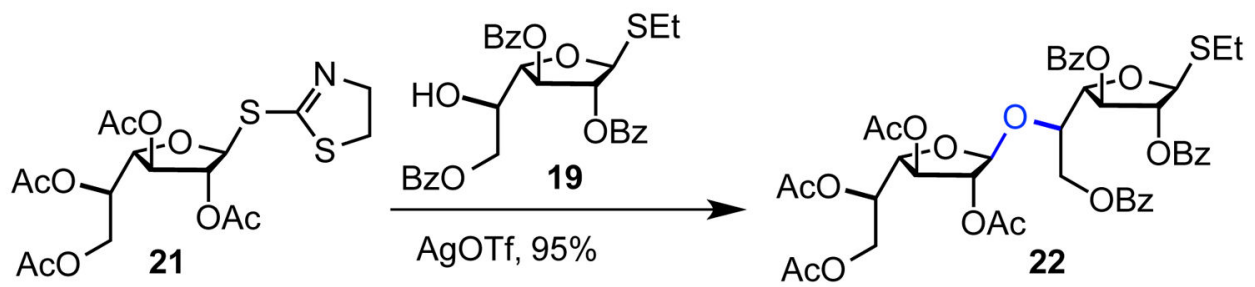

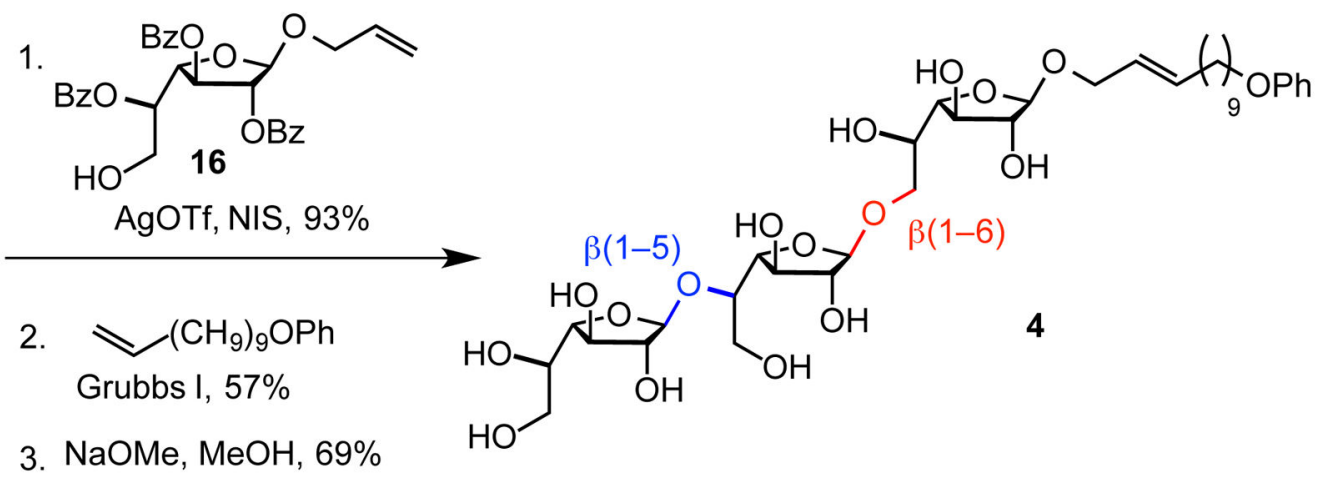

Scheme 2.

Synthesis of trisaccharide 4 

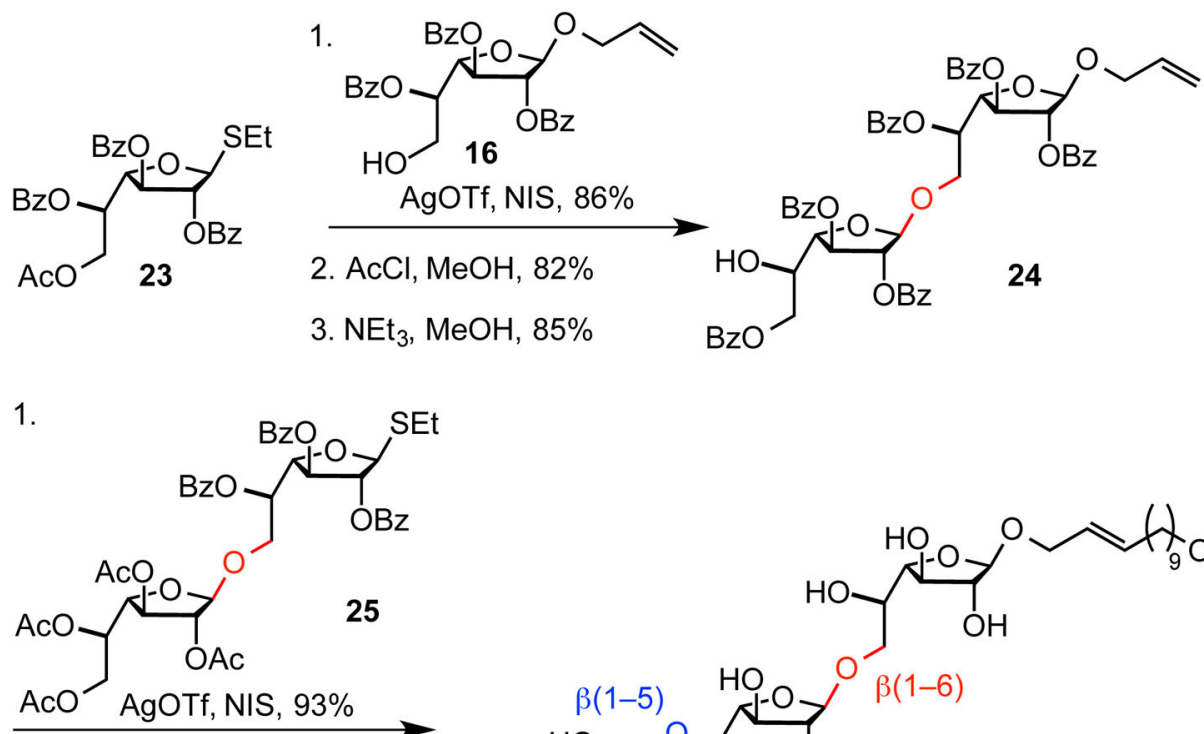

2. $\approx\left(\mathrm{CH}_{9}\right)_{9} \mathrm{OPh}$ Grubbs I, 75\%

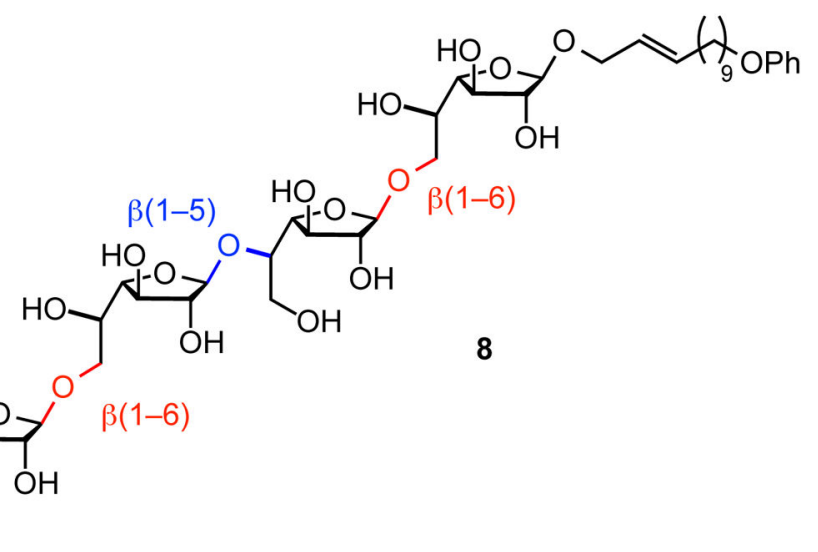

3. $\mathrm{NaOMe}, \mathrm{MeOH}, 98 \%$

Scheme 3.

Synthesis of tetrasaccharide 8 . 


\section{Table 1}

Apparent $K_{\mathrm{m}}$ and $k_{\text {cat }}$ for oligosaccharides 2 and $4-8$ determined by the continuous assay (Figure 6 ).

\begin{tabular}{|c|c|c|c|}
\hline Acceptor & $K_{\mathrm{m}}(\mu \mathrm{M})$ & $k_{\text {cat }}\left(\mathrm{s}^{-1}\right)$ & $k_{\text {cat }} / K_{\mathrm{m}}\left(10^{3} \mathrm{M}^{-1} \cdot \mathrm{s}^{-1}\right)$ \\
\hline $2 \mathrm{Gal} f \beta(1-6) \mathrm{Gal} f^{a}$ & $66 \pm 2$ & $1.0 \pm 0.03$ & $15 \pm 1$ \\
\hline $4 \mathrm{Gal} f(1-5) \mathrm{Gal} f(1-6) \mathrm{Gal} f$ & $13 \pm 2$ & $3.7 \pm 0.1$ & $280 \pm 20$ \\
\hline $5 \mathrm{Gal} f(1-6) \mathrm{Gal} f(1-5) \mathrm{Gal} f$ & $190 \pm 86$ & $0.72 \pm 0.15$ & $3.8 \pm 1.9$ \\
\hline $6 \mathrm{Gal} f(1-6) \mathrm{Gal} f(1-6) \mathrm{Gal} f$ & $34 \pm 16$ & $5.3 \pm 1.3$ & $160 \pm 80$ \\
\hline $7 \mathrm{Gal} f(1-5) \mathrm{Gal} f(1-5) \mathrm{Gal} f$ & $29 \pm 1$ & $0.87 \pm 0.01$ & $30 \pm 1$ \\
\hline 8 Tetrasaccharide & $13 \pm 1$ & $2.9 \pm 0.1$ & $220 \pm 20$ \\
\hline
\end{tabular}

${ }^{a}$ Data for compound 2 were obtained from reference 11. 